\title{
Events of Borel Sets, Construction of Borel Sets and Random Variables for Stochastic Finance
}

\author{
Peter Jaeger \\ Siegmund-Schacky-Str. 18a \\ 80993 Munich, Germany
}

\begin{abstract}
Summary. We consider special events of Borel sets with the aim to prove, that the set of the irrational numbers is an event of the Borel sets. The set of the natural numbers, the set of the integer numbers and the set of the rational numbers are countable, so we can use the literature [10 (pp. 78-81) as a basis for the similar construction of the proof. Next we prove, that different sets can construct the Borel sets 16. (pp. 9-10). Literature [16 (pp. 9-10) and 11] (pp. 11-12) gives an overview, that there exists some other sets for this construction. Last we define special functions as random variables for stochastic finance in discrete time. The relevant functions are implemented in the article 15], see 9 (p. 4). The aim is to construct events and random variables, which can easily be used with a probability measure. See as an example theorems (10) and (14) in [20. Then the formalization is more similar to the presentation used in the book 9. As a background, further literatures is [3] (pp. 9-12), [13] (pp. 17-20), and [8 (pp.32-35).
\end{abstract}

MSC: 28A05 03E30 03B35

Keywords: event; Borel set; random variable

MML identifier: FINANCE2, version: 8.1.03 5.26.1224

The notation and terminology used in this paper have been introduced in the following articles: [12], [15], [26], [4], [5], [27], 25], [1], [2], 6], [22], [19], [30], [20], [17], 24], [14], 29], [18, 223, 21], 28], and [7. 


\section{Events of Borel Sets}

Now we state the propositions:

(1) $1,-1 \in \mathbb{R}$.

(2) $e \in \mathbb{R} \backslash \mathbb{Q}$.

(3) $e \in \mathbb{R} \backslash \mathbb{Z}$.

(4) $e \in \mathbb{R} \backslash \mathbb{N}$

One can verify that $\mathbb{R} \backslash \mathbb{Q}$ is non empty and $\mathbb{R} \backslash \mathbb{Z}$ is non empty and $\mathbb{R} \backslash \mathbb{N}$ is non empty.

Now we state the propositions:

(5) Let us consider a real number $k$. Then $\{k\} \in$ the Borel sets.

(6) Let us consider real numbers $k_{1}, k_{2}$. Then $\left.] k_{1}, k_{2}\right]$ is an event of the Borel sets. The theorem is a consequence of (5).

The family of left closed half-lines yielding a family of subsets of $\mathbb{R}$ is defined by the term

(Def. 1) the set of all $[r,+\infty[$ where $r$ is an element of $\mathbb{R}$.

Now we state the propositions:

(7) Let us consider an extended real $E$. Then $\{E\}$ is a subset of $\overline{\mathbb{R}}$.

(8) Let us consider a set $Y$ and a natural number $k$. If $Y=\mathbb{R} \backslash\{k\}$, then $Y$ is an event of the Borel sets. The theorem is a consequence of (5).

Now we state the propositions:

(9) There exists a sequence $A$ of subsets of $\mathbb{N}$ such that for every natural number $n, A(n)=\{n\}$.

Proof: Define $\mathcal{U}$ (natural number) $=\left\{\$_{1}(\in \mathbb{N})\right\}$. For every element $x$ of $\mathbb{N}, \mathcal{U}(x) \in 2^{\mathbb{N}}$ by [7, (31)]. Consider $f$ being a sequence of subsets of $\mathbb{N}$ such that for every element $d$ of $\mathbb{N}, f(d)=\mathcal{U}(d)$ from [5, Sch. 8].

(10) Let us consider a sequence $A$ of subsets of $\mathbb{N}$. Suppose for every natural number $n, A(n)=\{n\}$. Let us consider a natural number $n$. Then (the partial unions of $A)(n) \in$ the Borel sets.

Proof: Define $\mathcal{J}$ [natural number] $\equiv$ (the partial unions of $A)\left(\$_{1}\right) \in$ the Borel sets. (The partial unions of $A)(0) \in$ the Borel sets. For every natural number $n$ such that $\mathcal{J}[n]$ holds $\mathcal{J}[n+1]$ by (5), [19, (3)]. For every natural number $n$, (the partial unions of $A)(n) \in$ the Borel sets.

(11) $\mathbb{R}$ is an event of the Borel sets. The theorem is a consequence of (5).

(12) $\mathbb{N}$ is an event of the Borel sets.

Proof: Consider $A$ being a sequence of subsets of the Borel sets such that for every natural number $n, A(n)=\{n\} . \cup A=\mathbb{N}$ by [19, (12)]. 
(13) $\mathbb{R} \backslash \mathbb{N}$ is an event of the Borel sets. The theorem is a consequence of (12).

(14) Let us consider a sequence $A_{1}$ of subsets of $\mathbb{R}$. Then there exists a sequence $A$ of subsets of $\mathbb{R}$ such that for every natural number $n, A(n)=$ (the partial unions of $\left.A_{1}\right)(n)$.

Proof: Define $\mathcal{U}$ (natural number) $=\left(\right.$ the partial unions of $\left.A_{1}\right)\left(\$_{1}\right)$. Consider $f$ being a sequence of subsets of $\mathbb{R}$ such that for every element $d$ of $\mathbb{N}, f(d)=\mathcal{U}(d)$ from [5, Sch. 4].

(15) $\mathbb{Z}$ is an event of the Borel sets.

Proof: Consider $A_{1}$ being a sequence of subsets of $\mathbb{R}$ such that for every natural number $n, A_{1}(n)=\{n\}$. Consider $A_{2}$ being a sequence of subsets of $\mathbb{R}$ such that for every natural number $n, A_{2}(n)=\{-n\}$. For every natural number $n$, (the partial unions of $\left.A_{1}\right)(n)$ is an event of the Borel sets by (5), [19, (21)]. Define $\mathcal{J}$ [natural number] $\equiv$ (the partial unions of $\left.A_{2}\right)\left(\$_{1}\right) \in$ the Borel sets. $\mathcal{J}[0]$. For every natural number $n$, (the partial unions of $\left.A_{2}\right)(n)$ is an event of the Borel sets by (5), [19, (21)]. Consider $B_{1}$ being a sequence of subsets of $\mathbb{R}$ such that for every natural number $n$, $B_{1}(n)=\left(\right.$ the partial unions of $\left.A_{1}\right)(n)$. For every natural number $n, B_{1}(n)$ is an event of the Borel sets. Consider $B_{2}$ being a sequence of subsets of $\mathbb{R}$ such that for every natural number $n, B_{2}(n)=$ (the partial unions of $\left.A_{2}\right)(n)$. For every natural number $n, B_{2}(n)$ is an event of the Borel sets. $\cup B_{1} \cup \cup B_{2}=\mathbb{Z}$ by [19, (12)], [30, (13)].

Let $k$ be a natural number and $p$ be an element of $\mathbb{R}$. The functor $\left\{\frac{p \cdot k}{n+1}\right\}_{n \in \mathbb{N}}$ yielding a sequence of subsets of $\mathbb{R}$ is defined by

(Def. 2) for every natural number $n$, it $(n)=\left\{p \cdot k \cdot(n+1)^{-1}\right\}$.

Note that the functor $\left\{\frac{p \cdot k}{n+1}\right\}_{n \in \mathbb{N}}$ yields a sequence of subsets of the Borel sets. One can check that $\left\{\frac{p \cdot k}{n+1}\right\}_{n \in \mathbb{N}}$ is (the Borel sets)-valued.

Now we state the proposition:

(16) Let us consider an element $p$ of $\mathbb{R}$ and a natural number $k$. If $k>0$ and $p \neq 0$, then $\left\{\frac{p \cdot k}{n+1}\right\}_{n \in \mathbb{N}}$ is one-to-one.

Let $k$ be a natural number and $p$ be an element of $\mathbb{R}$.

The functor $\left(\bigcup_{i=0}^{n}\left\{\frac{p \cdot k}{i+1}\right\}\right)_{n \in \mathbb{N}}$ yielding a sequence of subsets of $\mathbb{R}$ is defined by

(Def. 3) $i t(0)=\left(\left\{\frac{p \cdot k}{n+1}\right\}_{n \in \mathbb{N}}\right)(0)$ and for every natural number $n$, it $(n+1)=$ $i t(n) \cup\left(\left\{\frac{p \cdot k}{n+1}\right\}_{n \in \mathbb{N}}\right)(n+1)$.

Note that the functor $\left(\bigcup_{i=0}^{n}\left\{\frac{p \cdot k}{i+1}\right\}\right)_{n \in \mathbb{N}}$ yields a sequence of subsets of the Borel sets. One can verify that $\left(\bigcup_{i=0}^{n}\left\{\frac{p \cdot k}{i+1}\right\}\right)_{n \in \mathbb{N}}$ is (the Borel sets)-valued and $\left(\bigcup_{i=0}^{n}\left\{\frac{p \cdot k}{i+1}\right\}\right)_{n \in \mathbb{N}}$ is non descending. 
The functor $\left\{\frac{p \cdot k}{n+1}: k, n \in \mathbb{N}\right\}$ yielding a sequence of subsets of $\mathbb{R}$ is defined by

(Def. 4) $i t(0)=\bigcup\left(\left(\bigcup_{i=0}^{n}\left\{\frac{p \cdot 0}{i+1}\right\}\right)_{n \in \mathbb{N}}\right)$ and for every natural number $n$, it $(n+1)=$ $i t(n) \cup \bigcup\left(\left(\bigcup_{i=0}^{n}\left\{\frac{p \cdot(n+1)}{i+1}\right\}\right)_{n \in \mathbb{N}}\right)$.

One can check that the functor $\left\{\frac{p \cdot k}{n+1}: k, n \in \mathbb{N}\right\}$ yields a sequence of subsets of the Borel sets. Let us note that $\left\{\frac{p \cdot k}{n+1}: k, n \in \mathbb{N}\right\}$ is (the Borel sets)-valued and $\left\{\frac{p \cdot k}{n+1}: k, n \in \mathbb{N}\right\}$ is non descending.

Now we state the propositions:

(17) Let us consider elements $a, b$ of $\mathbb{R}$. Suppose $a=1$ and $b=-1$. Then $\bigcup\left\{\frac{a \cdot k}{n+1}: k, n \in \mathbb{N}\right\} \cup \bigcup\left\{\frac{b \cdot k}{n+1}: k, n \in \mathbb{N}\right\}=\mathbb{Q}$.

ProOF: For every object $x, x \in \bigcup\left\{\frac{a \cdot k}{n+1}: k, n \in \mathbb{N}\right\} \cup \bigcup\left\{\frac{b \cdot k}{n+1}: k, n \in \mathbb{N}\right\}$ iff $x \in \mathbb{Q}$ by [19, (12)], [1, (6)], [17, (8)], [27, (2)].

(18) $\mathbb{Q}$ is an event of the Borel sets. The theorem is a consequence of (1) and (17).

(19) $\mathbb{R} \backslash \mathbb{Z}$ is an event of the Borel sets. The theorem is a consequence of (15).

(20) $\mathbb{R} \backslash \mathbb{Q}$ is an event of the Borel sets. The theorem is a consequence of (18).

(21) $\mathbb{I} \mathbb{Q}$ is an event of the Borel sets. The theorem is a consequence of (18).

\section{Construction of Borel Sets}

Now we state the proposition:

(22) The Borel sets $=\sigma$ (the family of left closed half-lines).

Proof: $\sigma$ (the family of left closed half-lines) $\subseteq \sigma$ (Halflines) by [15, (3)]. $\sigma$ (Halflines) $\subseteq \sigma$ (the family of left closed half-lines) by [15, (2)], [19, (15)].

\section{Random Variables for Stochastic Finance in Discrete Time}

In the sequel $\Omega$ denotes non empty set, $\Sigma$ denotes a $\sigma$-field of subsets of $\Omega$, and $X, Y, Z$ denote functions from $\Omega$ into $\mathbb{R}$.

Let us consider random variables $X, Y$ of $\Sigma$ and the Borel sets. Now we state the propositions:

(23) $X+Y$ is a random variable of $\Sigma$ and the Borel sets.

(24) $X-Y$ is a random variable of $\Sigma$ and the Borel sets.

(25) $X \cdot Y$ is a random variable of $\Sigma$ and the Borel sets.

Now we state the proposition: 
(26) Let us consider a real number $r$ and a random variable $X$ of $\Sigma$ and the Borel sets. Then $r \cdot X$ is a random variable of $\Sigma$ and the Borel sets.

Let $\Omega, \Omega_{1}$ be non empty sets, $F$ be a $\sigma$-field of subsets of $\Omega, F_{1}$ be a $\sigma$-field of subsets of $\Omega_{1}$, and $X$ be a set. Assume $X=$ the set of random variables on $F$ and $F_{1}$. Let $k$ be an element of $X$. Let us observe that the change element to function $F, F_{1}$, and $k$ yields a random variable of $F$ and $F_{1}$. Let $\Omega$ be a non empty set and $X$ be a non empty set. Let us note that the random variables for future elements of portfolio value of $F$ and $k$ yields a random variable of $F$ and the Borel sets. Let $p_{1}$ be a natural number, $\Omega, \Omega_{1}$ be non empty sets, $F_{1}$ be a $\sigma$-field of subsets of $\Omega_{1}$, and $X$ be a set. Assume $X=$ the set of random variables on $F$ and $F_{1}$. Let $G$ be a sequence of $X$. Let us note that the element of $F, F_{1}, G$, and $p_{1}$ yields a random variable of $F$ and $F_{1}$. Let $\Omega$ be a non empty set, $X$ be a non empty set, $\varphi$ be a sequence of real numbers, and $n$ be a natural number. The random variables for the future elements of portfolio value of $(\varphi, F, G, n)$ yielding a function from $\Omega$ into $\mathbb{R}$ is defined by

(Def. 5) for every element $w$ of $\Omega$, it $(w)=$ (the random variables for future elements of portfolio value of $F$ and $G(n))(w) \cdot \varphi(n)$.

One can verify that the random variables for the future elements of portfolio value of $(\varphi, F, G, n)$ yields a random variable of $F$ and the Borel sets. Let $w$ be an element of $\Omega$. The elements of the random variables for the future elements of portfolio value of $(\varphi, F, G, w)$ yielding a sequence of real numbers is defined by

(Def. 6) for every natural number $n$, it $(n)=$ (the random variables for the future elements of portfolio value of $(\varphi, F, G, n))(w)$.

Let $d$ be a natural number. Let us note that the portfolio value for future extension of $d, \varphi, F, G$, and $w$ yields a real number and is defined by the term

(Def. 7$) \quad\left(\sum_{\alpha=0}^{\kappa}\right.$ (the elements of the random variables for the future elements of portfolio value of $(\varphi, F, G, w))(\alpha))_{\kappa \in \mathbb{N}}(d)$.

\section{REFERENCES}

[1] Grzegorz Bancerek. The fundamental properties of natural numbers Formalized Mathematics, 1(1):41-46, 1990.

[2] Grzegorz Bancerek. The ordinal numbers. Formalized Mathematics, 1(1):91-96, 1990.

[3] Siegfried Bosch. Lineare Algebra. Springer, Berlin, Heidelberg, 4 edition, 2008.

[4] Czesław Byliński. Functions and their basic properties Formalized Mathematics, 1(1): 55-65, 1990.

[5] Czesław Byliński. Functions from a set to a set Formalized Mathematics, 1(1):153-164, 1990.

[6] Czesław Byliński. Partial functions. Formalized Mathematics, 1(2):357-367, 1990.

[7] Czesław Byliński. Some basic properties of sets Formalized Mathematics, 1(1):47-53, 1990. 
[8] Gerd Fischer. Lineare Algebra. Vieweg, Braunschweig, Wiesbaden, 13 edition, 2002.

[9] Hans Föllmer and Alexander Schied. Stochastic Finance: An Introduction in Discrete Time, volume 27 of Studies in Mathematics. de Gruyter, Berlin, 2nd edition, 2004.

[10] Otto Forster. Analysis 1. Vieweg-Verlag, Braunschweig/Wiesbaden, 6th edition, 2001.

[11] Hans-Otto Georgii. Stochastik, Einführung in die Wahrscheinlichkeitstheorie und Statistik. deGruyter, Berlin, 2nd edition, 2004.

[12] Adam Grabowski. On the subcontinua of a real line Formalized Mathematics, 11(3): 313-322, 2003.

[13] Harro Heuser. Lehrbuch der Analysis. Teil 1. Teubner, Stuttgart, Leipzig, Wiesbaden, 15 edition, 2003.

[14] Krzysztof Hryniewiecki. Basic properties of real numbers Formalized Mathematics, 1(1): 35-40, 1990.

[15] Peter Jaeger. Elementary introduction to stochastic finance in discrete time. Formalized Mathematics, 20(1):1-5, 2012. doi 10.2478/v10037-012-0001-5.

[16] Achim Klenke. Wahrscheinlichkeitstheorie. Springer-Verlag, Berlin, Heidelberg, 2006.

[17] Andrzej Kondracki. Basic properties of rational numbers. Formalized Mathematics, 1(5): 841-845, 1990.

[18] Jarosław Kotowicz. Convergent real sequences. Upper and lower bound of sets of real numbers Formalized Mathematıcs, 1(3):477-481, 1990.

[19] Andrzej Nędzusiak. $\sigma$-fields and probability. Formalized Mathematics, 1(2):401-407, 1990.

[20] Hiroyuki Okazaki and Yasunari Shidama. Random variables and product of probability spaces. Formalized Mathematics, 21(1):33-39, 2013. doi 10.2478/forma-2013-0003.

[21] Beata Padlewska. Families of sets Formalized Mathematics, 1(1):147-152, 1990.

[22] Konrad Raczkowski and Andrzej Nędzusiak. Real exponents and logarithms Formalized Mathematics, 2(2):213-216, 1991.

[23] Konrad Raczkowski and Andrzej Nędzusiak. Series. Formalized Mathematics, 2(4):449452, 1991.

[24] Konrad Raczkowski and Paweł Sadowski. Topological properties of subsets in real numbers Formalized Mathematics, 1(4):777-780, 1990.

[25] Andrzej Trybulec. On the sets inhabited by numbers. Formalized Mathematics, 11(4): 341-347, 2003.

[26] Andrzej Trybulec and Agata Darmochwał. Boolean domains. Formalized Mathematics, 1 (1):187-190, 1990.

[27] Michał J. Trybulec. Integers Formalized Mathematics, 1(3):501-505, 1990.

[28] Zinaida Trybulec. Properties of subsets Formalized Mathematics, 1(1):67-71, 1990.

[29] Edmund Woronowicz. Relations and their basic properties. Formalized Mathematics, 1 (1):73-83, 1990.

[30] Bo Zhang, Hiroshi Yamazaki, and Yatsuka Nakamura. Set sequences and monotone class. Formalized Mathematics, 13(4):435-441, 2005. 